\title{
TORUS ACTIONS ON ORIENTED MANIFOLDS OF GENERALIZED ODD TYPE
}

\author{
DONGHOON JANG
}

\begin{abstract}
In [LS], Landweber and Stong prove that if a closed spin manifold $M$ admits a smooth $S^{1}$-action of odd type, then its signature $\operatorname{sign}(M)$ vanishes. In this paper, we extend the result to a torus action on a closed oriented manifold with generalized odd type.
\end{abstract}

\section{INTRODUCTION}

The existence of a non-trivial group action on a manifold gives certain restrictions on the manifold and one of them is a characteristic class. Atiyah and Hirzebruch prove that if a closed spin manifold admits a non-trivial circle action, then its $\hat{A}$-genus vanishes [AH]. Hattori generalizes the result to $\operatorname{spin}^{c}$-manifolds [Ha]. It is shown later that $\hat{A}$-genus vanishes if an oriented manifold with finite second and fourth homotopy groups admits an $S^{1}$-action [HH], [HH3].

In this paper, we discuss the vanishing of $L$-genus of an oriented manifold, that is, the signature of the manifold. The $L$-genus is the characteristic class belonging to the power series $f(x)=\frac{\sqrt{x}}{\tanh \sqrt{x}}$. The signature of an oriented manifold $M$ is the index of the signature operator on $M$. The AtiyahSinger index theorem states that the $L$-genus of an oriented manifold $M$ is equal to the signature of $M$ [AS]. Kawakubo and Uchida prove that if a closed oriented manifold $M$ admits a semi-free $S^{1}$-action with $\operatorname{dim}\left(M^{S^{1}}\right)<$ $\frac{1}{2} \operatorname{dim} M$, then the signature of $M$ vanishes [KU]. Li and Liu generalize the result to a so-called prime action [LL]. For a vanishing result on the signature of a manifold with a finite group action, see $[\mathrm{E}]$ for instance.

Let $M$ be an orientable manifold. Introduce a Riemannian metric on $M$. A spin structure on $M$ is an equivariant lift $P$ (called a principal $\operatorname{Spin}(n)$ bundle) of the oriented orthonormal frame bundle $Q$ (called the principal $S O(n)$-bundle) over $M$ with respect to the double covering $\pi: \operatorname{Spin}(n) \rightarrow$ $S O(n)$.

Let the circle act on a spin manifold $M$. Then the action lifts to an action on the principal $S O(n)$-bundle $Q$. The action is called of even type, if it

Donghoon Jang is supported by Basic Science Research Program through the National Research Foundation of Korea (NRF) funded by the Ministry of Education (2018R1D1A1B07049511). 
further lifts to an action on the principal $\operatorname{Spin}(n)$-bundle $P$. The action is called of odd type, if it fails to lift to an action on $P$.

Given an action of a Lie group $G$ on a manifold $M$, denote $M^{G}$ by the set of points fixed by the $G$-action on $M$, i.e., $M^{G}=\{p \in M \mid g \cdot p=p, \forall g \in G\}$. If $H$ is a subgroup of $G$, then define the set $M^{H}$ of points fixed by the $H$-action in the same way.

Given a circle action on a spin manifold $M$, as a subgroup of $S^{1}, \mathbb{Z}_{2}$ also acts on $M$. The $S^{1}$-action on $M$ is of even type if and only if each connected component of the set $M^{\mathbb{Z}_{2}}$ has codimension congruent to 0 modulo 4. Similarly, the $S^{1}$-action on $M$ is of odd type if and only if each connected component of $M^{\mathbb{Z}_{2}}$ has codimension congruent to 2 modulo 4 . For this, see [AH].

Now consider a circle action on a manifold $M$. Since $M$ need not allow a spin structure, we use the latter equivalent definition to define an action of even type and an action of odd type. The $S^{1}$-action is called of even type, if each connected component of $M^{\mathbb{Z}_{2}}$ has codimension congruent to 0 modulo 4 and of odd type, if each connected component of $M^{\mathbb{Z}_{2}}$ has codimension congruent to 2 modulo 4 . As before, $\mathbb{Z}_{2}$ acts on $M$ as a subgroup of $S^{1}$. In [HH2], H. Herrera and R. Herrera adapt these alternative definitions for circle actions on oriented manifolds.

Landweber and Stong prove that a closed spin manifold admitting a circle action of odd type must have vanishing $L$-genus [LS].

Theorem 1.1. [LS] If a closed spin manifold $M$ admits a smooth $S^{1}$-action of odd type, then its signature $\operatorname{sign}(M)$ vanishes.

In this paper, we generalize Theorem 1.1 in three directions:

(1) from spin manifolds to oriented manifolds.

(2) from circle actions to torus actions.

(3) from odd type to generalized odd type.

In this paper, for a torus action on a manifold, we introduce the notion of an action of generalized odd type.

Definition 1.2. Let a $k$-torus $\mathbb{T}^{k}$ act on a manifold $M$. Let $S$ be a closed subgroup of $\mathbb{T}^{k}$.

(1) The $\mathbb{T}^{k}$-action is called of even type with respect to $S$, if for any connected component $Z$ of $M^{S}$, we have $\operatorname{dim} Z \equiv \operatorname{dim} M \bmod 4 . A n$ action of a torus $\mathbb{T}^{k}$ on a manifold is called of generalized even type, if it is of even type with respect to some closed subgroup of $\mathbb{T}^{k}$.

(2) The $\mathbb{T}^{k}$-action is called of odd type with respect to $S$, if for any connected component $Z$ of $M^{S}$, we have $\operatorname{dim} Z \equiv \operatorname{dim} M-2 \bmod 4$. An action of a torus $\mathbb{T}^{k}$ on a manifold is called of generalized odd type, if it is of odd type with respect to some closed subgroup of $\mathbb{T}^{k}$.

Therefore, a circle action on a manifold being of odd type is a special of a torus action of generalized odd type where the torus is the circle, and the closed subgroup is $\mathbb{Z}_{2}$. The main result of this paper is the following: 
TORUS ACTIONS ON ORIENTED MANIFOLDS OF GENERALIZED ODD TYPE 3

Theorem 1.3. Let a torus act on a closed oriented manifold $M$. If the action is of generalized odd type, then the signature of $M$ vanishes.

In [HH2], H. Herrera and R. Herrera prove vanishing results on characteristic classes of manifolds admitting circle actions. One of them is that if a $4 n$-dimensional oriented manifold with finite second homotopy group admits a circle action of odd type, then its signature vanishes. As a special case of Theorem 1.3 where a torus group is the circle group and the closed subgroup is $\mathbb{Z}_{2}$, we recover the result without the assumption on the second homotopy group of the manifold.

\section{Preliminaries And the Proof of the main Result}

Let the circle act on a closed oriented manifold $M$. Then the equivariant index of the signature operator is defined for each element of $S^{1}$. In [AS], it is proved that the equivariant index is rigid under the circle action, i.e., is independent of the choice of an element of $S^{1}$, and is equal to the signature of $M$. Consequently, the signature of $M$ is equal to the sum of the signatures of the connected components of $M^{S^{1}}$.

Now, let a $k$-torus $\mathbb{T}^{k}$ act on a closed oriented manifold $M$. Then there exists a circle $S^{1}$ inside $\mathbb{T}^{k}$ that has the same fixed point set as $\mathbb{T}^{k}$, i.e., $M^{S^{1}}=M^{\mathbb{T}^{k}}$. As for $S^{1}$-actions, for an action of a torus the signature of $M$ is equal to the sum of the signatures of the connected components of $M^{\mathbb{T}^{k}}$. It follows from Theorem 6.12 in [AS] and is stated explicitly in [KR].

Theorem 2.1. [AS], [KR] Let a $k$-torus $\mathbb{T}^{k}$ act on a closed oriented manifold $M$. Then $\operatorname{sign}(M)=\operatorname{sign}\left(M^{\mathbb{T}^{k}}\right)$.

By $\operatorname{sign}\left(M^{\mathbb{T}^{k}}\right)$, it means the sum of the signatures of all connected components of $M^{\mathbb{T}^{k}}$, i.e., $\operatorname{sign}\left(M^{\mathbb{T}^{k}}\right)=\sum_{N \subset M^{\mathbb{T}^{k}}} \operatorname{sign}(N)$.

In $[\mathrm{Ko}]$, Kobayashi proves that the fixed point set of a torus action on an orientable manifold is orientable.

Lemma 2.2. [Ko] Let a torus act on an orientable manifold $M$. Then the fixed point set is a union of closed orientable manifolds.

Given a circle action on an oriented manifold, H. Herrera and R. Herrera prove the orientability of the set of points fixed by a subgroup of $S^{1}$.

Lemma 2.3. [HH] Let $M$ be an oriented, 2n-dimensional, smooth manifold endowed with a smooth $S^{1}$-action. Consider $\mathbb{Z}_{k} \subset S^{1}$ and its corresponding action on $M$. If $k$ is odd then the fixed point set $M^{\mathbb{Z}_{k}}$ of the $\mathbb{Z}_{k}$-action is orientable. If $k$ is even and a connected component $Z$ of $M^{\mathbb{Z}_{k}}$ contains a fixed point of the $S^{1}$-action, then $Z$ is orientable.

Let $S$ be a closed subgroup of $\mathbb{T}^{k}$. Then $S$ is Lie isomorphic to a product of $S^{1}$ 's and $\mathbb{Z}_{a}$ 's, i.e., $S \approx S^{1} \times \cdots \times S^{1} \times \mathbb{Z}_{a_{1}} \times \cdots \times \mathbb{Z}_{a_{m}}$, where $a_{i}$ 's are positive integers bigger than 1 . Note that the $a_{i}$ 's may have repeated 
elements. By using Lemma 2.2 and Lemma 2.3, we extend Lemma 2.3 to torus actions.

Lemma 2.4. Let a $k$-torus $\mathbb{T}^{k}$ act on a $2 n$-dimensional orientable manifold $M$ and $S$ a closed subgroup of $\mathbb{T}^{k}$. Let $Z$ be a connected component of $M^{S}$. If $Z$ contains a $\mathbb{T}^{k}$-fixed point (i.e., if $Z \cap M^{\mathbb{T}^{k}} \neq \emptyset$ ), then $Z$ is orientable.

Proof. Without loss of generality, by choosing an orientation, assume that $M$ is oriented. Since $S$ is a closed subgroup of $\mathbb{T}^{k}, S$ is isomorphic to

$$
S=\left(S^{1}\right)^{l} \times \mathbb{Z}_{a_{1}} \times \cdots \times \mathbb{Z}_{a_{m}}
$$

for some $l \geq 0$ and positive integers $a_{i}>1$ for $i=1, \cdots, m$.

Denote $S_{i}=\left(S^{1}\right)^{l} \times \mathbb{Z}_{a_{1}} \times \cdots \times \mathbb{Z}_{a_{i}}$ for $i=0,1, \cdots, m$. Also, denote $M_{i}$ by the set of points fixed by $S_{i}$-action, i.e., $M_{i}=M^{S_{i}}$. We prove that for any $i$ if $Z_{i}$ is a connected component of $M_{i}$ that contains $Z$, then $Z_{i}$ is orientable.

Consider the case that $i=0$. Then $S_{0}=\left(S^{1}\right)^{l}$. By Lemma 2.2, the set $M^{S_{0}}=M^{\left(S^{1}\right)^{l}}$ of points fixed by the $\left(S^{1}\right)^{l}$-action is a union of smaller dimensional closed orientable manifolds.

Suppose that a connected component $Z_{i-1}$ of $M_{i-1}$ is orientable and contains $Z$. On $Z_{i-1}$, we have an induced action of $G_{i}=\mathbb{T}^{k} / S_{i}=\left(S^{1}\right)^{k} /\left(S^{1}\right)^{l} \times$ $\mathbb{Z}_{a_{1}} \times \cdots \times \mathbb{Z}_{a_{i}}=\left(S^{1}\right)^{k-l}=\mathbb{T}^{k-l}$. Moreover, as a subgroup of $G_{i}, \mathbb{Z}_{a_{i}}$ acts on $Z_{i-1}$. Given a generator $b_{i}$ of $\mathbb{Z}_{a_{i}}$, there exists $X_{i}$ in the Lie algebra of $\mathbb{T}^{k-l}$ such that $\exp \left(\frac{1}{a_{i}} X_{i}\right)=b_{i}$. In other words, $X_{i}$ generates a circle $S^{1}$ such that $\mathbb{Z}_{a_{i}} \subset S^{1}$. Denote the circle by $H_{i}$.

Therefore, on the orientable manifold $Z_{i-1}$, we have the action of the circle $H_{i}$ and the action of $\mathbb{Z}_{a_{i}}$, as a subgroup of $H_{i}$. A connected component $Z_{i}$, the set of points in $Z_{i-1}$ that are fixed by the $\mathbb{Z}_{a_{i}}$-action and contains $Z$, contains a $H_{i}$-fixed point, since it contains $Z$ which is fixed by the $\mathbb{T}^{k}$-action and $H_{i}$ is a subgroup of $\mathbb{T}^{k}$.

Apply Lemma 2.3 for the action of the circle $H_{i}$ on $Z_{i-1}$ with its subgroup $\mathbb{Z}_{a_{i}}$. Since the connected component $Z_{i}$ of $Z_{i-1}^{\mathbb{Z}_{a_{i}}}$ contains a $H_{i}$-fixed point, it follows that $Z_{i}$ is orientable.

Note that $Z_{m}=Z$. The lemma then follows by inductive argument.

Note that in Lemma 2.4, we can remove the condition that $Z$ contains a $\mathbb{T}^{k}$-fixed point, if all $a_{i}$ are odd. With Lemma 2.4, we are ready to prove our main result.

Proof of Theorem 1.3. If the dimension of $M$ is not divisible by 4 , then the signature of $M$ is defined to be 0 and hence the theorem follows. Therefore, from now on, suppose that the dimension of $M$ is divisible by 4 .

Let $\mathbb{T}^{k}$ be the torus which acts on $M$. Let $S$ be the subgroup of the torus $\mathbb{T}^{k}$ such that the torus action is of odd type with respect to $S$. Let $Z$ be a connected component of the set $M^{S}$ of points fixed by the $S$-action on 
$M$ that contains a $\mathbb{T}^{k}$-fixed point, i.e., $Z \cap M^{\mathbb{T}^{k}} \neq \emptyset$. Then by Lemma 2.4, $Z$ is orientable. Choose an orientation of $Z$. Since the $\mathbb{T}^{k}$-action is of odd type with respect to the closed subgroup $S, \operatorname{dim} Z \equiv \operatorname{dim} M-2 \bmod 4$. Since $\operatorname{dim} M \equiv 0 \bmod 4$, we have that $\operatorname{dim} Z \equiv 2 \bmod 4$. Therefore, we have that $\operatorname{sign}(Z)=0$. On $Z$, there is an induced action of $\mathbb{T}^{k} / S=\mathbb{T}^{k^{\prime}}$. Moreover, the set of points in $Z$ that are fixed by the induced $\mathbb{T}^{k^{\prime}}$-action is precisely the set of points in $Z$ that are fixed by the entire $\mathbb{T}^{k}$-action on $M$, i.e., $Z^{\mathbb{T}^{k^{\prime}}}=Z \cap M^{\mathbb{T}^{k}}$. Applying Theorem 2.1 to the induced action of the torus $\mathbb{T}^{k^{\prime}}=\mathbb{T}^{k} / S$ on $Z$, we have that

$$
\sum_{N \subset Z^{\mathbb{T}^{k^{\prime}}}} \operatorname{sign}(N)=\operatorname{sign}(Z)=0 .
$$

On the other hand, by directly applying Theorem 2.1 to the action of the $k$-torus $\mathbb{T}^{k}$ on $M$, we have that

$$
\operatorname{sign}(M)=\sum_{N \subset M^{\mathbb{T}} k} \operatorname{sign}(N)
$$

Since $M^{\mathbb{T}^{k}} \subset M^{S} \subset M$, each connected component $N$ of $M^{\mathbb{T}^{k}}$ is contained in a unique connected component $Z$ of $M^{S}$ which contains a $\mathbb{T}^{k}$-fixed point, as $Z$ contains the $\mathbb{T}^{k}$-fixed component $N$. Therefore, we have that

$$
\begin{aligned}
& \operatorname{sign}(M)=\sum_{N \subset M^{\mathbb{T}} k} \operatorname{sign}(N)=\sum_{Z \subset M^{S}, Z \cap M^{\mathbb{T}^{k}} \neq \emptyset} \sum_{N \subset Z \cap M^{\mathbb{T}} k} \operatorname{sign}(N) \\
& =\sum_{Z \subset M^{S}, Z \cap M^{\mathbb{T}^{k}} \neq \emptyset} \operatorname{sign}(Z)=0 .
\end{aligned}
$$

\section{REFERENCES}

[AH] M. Atiyah and F. Hirzebruch: Spin manifolds and group actions. Essays on Topology and Related Topics, Berlin-Heidelberg, Springer (1969) 18-28.

[AS] M. Atiyah and I. Singer: The index of elliptic operators: III. Ann. Math. 87 (1968) 546-604.

[E] A. Edmonds: Orientability of fixed point sets. P. Amer. Math. Soc. 82 (1981) 120-124.

[Ha] A. Hattori: Spin ${ }^{c}$-structures and $S^{1}$-actions. Invent. Math. 48 (1978) 7-32.

[HH] H. Herrara and R. Herrera: Â-genus on non-spin manifolds with $S^{1}$ actions and the classification of positive quaternion-Kähler 12-manifolds. J. Differential Geometry. 61 (2002) 341-364.

[HH2] Haydee Herrera and Rafael Herrera: The signature and the elliptic genus of $\pi_{2}$ finite manifolds with circle actions. Topology Appl. 136 (2004) 251-259.

[HH3] H. Herrara and R. Herrera: Erratum to "Â-genus on non-spin manifolds with $S^{1}$ actions and the classification of positive quaternion-Kähler 12-manifolds." J. Differential Geom. 90 (2012) no. 3, 521.

[Ko] S. Kobayashi: Fixed points of isometries. Nagoya Math. J. 13 (1958) 63-68. 
[KR] K. Kawakubo and F. Raymond: The Index of Manifolds with Toral Actions and Geometric Interpretations of the $\sigma\left(\infty,\left(S^{1}, M^{n}\right)\right)$ Invariant of Atiyah and Singer. Inventiones Math. 15 (1972) 53-66.

[KU] K. Kawakubo and F. Uchida: On the index of a semi-free $S^{1}$-action. J. Math. Soc. Japan. 23 (1971) 351-355.

[LS] P. Landweber and R. Stong: Circle actions on spin manifolds and characteristic numbers. Topology 27 (1988) No.2, 145-161.

[LL] P. Li and K. Liu: Circle actions and some vanishing results on manifolds. Int. J. Math. 22 (2011) 1603-1610.

Department of Mathematics, Pusan National University, Pusan, Korea

E-mail address: donghoonjang@pusan.ac.kr 